\title{
Parámetros del Habla en Adultos Normales Chilenos
}

\author{
Parameters of Speech in Normal \\ Chilean Adult
}

Lilian Toledo R.

Académico

Escuela de Fonoaudiología Universidad de Chile

\section{Claudia Bahamonde D.}

Fonoaudióloga

Universidad de Chile

Javiera González J.

Fonoaudióloga

Universidad de Chile

\section{Macarena Martínez 0. \\ Académico \\ Escuela de Fonoaudiología \\ Universidad de Chile}

María M. Muñoz M.

Fonoaudióloga

Universidad de Chile

\section{Daniel Muñoz S.}

Médico

Programa de Magíster en Bioestadística

Universidad de Chile

Contacto con el autor:

Lilian Toledo R. (Mg)

Escuela de Fonoaudiología

Universidad de Chile

Av. Independencia 1027 Santiago, Chile

Tel: (56 2) 9786181

Fax: (56 2) 9786608

e-mail: Itoledo@med.uchile.cl

Recibido: Agosto, 2010

Aceptado: Abril, 2011

\section{RESUMEN}

El propósito de este estudio fue comparar el rendimiento en tareas de habla de adultos chilenos según edad y género, para obtener datos normativos destinados a la evaluación del habla en nuestra población. Se evaluó 90 sujetos entre 40-69 años, con un protocolo que incluye: Tiempo Máximo Fonatorio (TMF), movimientos orales mandibulares, labiales y linguales, diadococinesias orales, velocidad de lectura oral y velocidad del habla automática. Al comparar por género, los hombres rinden significativamente mejor que las mujeres en TMF, movimientos linguales, repetición de sílabas y palabras. La comparación por edad arroja diferencias en movimientos labiales, repetición de sílabas, palabras y velocidad de habla automática, con un rendimiento superior entre 50-59 años. Basado en estos hallazgos, este estudio revela que el rendimiento en tareas de habla presenta diferencias según la edad y género de los sujetos. Los valores obtenidos difieren a los reportados en estudios de habla inglesa.

Palabras clave: habla en adultos chilenos, diadococinesias orales, tiempo máximo fonatorio.

\section{ABSTRACT}

The aim of this study was to compare the performance in speech tasks of Chilean adults according to age and gender in order to obtain normative data for the assessment of speech in the Chilean population. A total of 90 subjects aged between 40 and 69 years were evaluated. The test battery was comprised of measures of Maximum Phonation Duration (MPD), oral, jaw, lips and lingual movements, oral diadochokinesis, oral reading speed and automatic speech speed. Male subjects performed significantly better than female subjects in MPD, tongue movements, and repetition of syllables and words. Adults aged 50-59 years performed significantly better than the other age groups in lips movements, repetition of syllables and words, and automatic speech speed. Based on these findings this study indicates that the performance in speech tasks differs according to the age and gender of the subjects. The values obtained in this study differ from those obtained in other studies of English-speaking subjects.

Keywords: speech in Chilean adults, oral diadochokinesis, maximum phonation duration. 


\section{Introducción}

El habla representa una amplia área de estudio de la fonoaudiología, debido a que cumple una función fundamental dentro del proceso de la comunicación oral.

Su producción requiere de la participación conjunta de los niveles físico, acústico, aerodinámico, neuromuscular y cinemático ${ }^{1}$, los cuales poseen un correlato biológico sustentado principalmente en mecanismos neurológicos superiores, destacando entre ellos la corteza cerebral, núcleos de la base, tronco encefálico, cerebelo y médula espinal.

El habla constituye un indicador sensible del estado funcional de estos sistemas, de ahí la importancia de la detección oportuna de sus posibles alteraciones, ya que cambios en el habla pueden indicar la presencia de un trastorno neurológico².

Las alteraciones motoras del habla corresponden al trastorno de la comunicación más frecuente, alcanzando una proporción del $41 \%$ del total de los trastornos adquiridos de la comunicación en un estudio longitudinal realizado en la Clínica Mayo².

En Chile, la principal etiología en este tipo de trastorno son los accidentes cerebrovasculares (ACV), que corresponden a la segunda causa de muerte y la primera causa de hospitalización en sujetos mayores de 65 años de edad, y cuya tasa es de 168,4 casos por 100.000 habitantes, con lo que se esperan aproximadamente 27.000 casos nuevos al año, de los cuales un $50 \%$ presentará un trastorno de habla ${ }^{3,4}$.

Dado lo anterior, es imprescindible disponer de instrumentos de evaluación que cuenten con adecuados criterios de normalidad, siendo especialmente valiosos aquéllos derivados del soporte empírico. De esta forma, la distinción entre habla normal y habla patológica se sustentará bajo juicios más objetivos para ser aplicados en la práctica clínica.

Actualmente en nuestro país no disponemos de valores normativos de parámetros de habla, que se evalúan con frecuencia en la clínica, utilizándose hasta el momento aquéllos obtenidos en el extranjero a partir de la lengua inglesa.

Es por esto que surge la necesidad de contar con instrumentos de evaluación de la producción del habla que se encuentren estandarizados bajo datos obtenidos en la población de Chile.

Por otro lado, resulta interesante conocer el rendimiento de los sujetos de acuerdo a características tales como la edad y el género, ya que son variables que otros estudios han indicado que influyen en el rendimiento frente a tareas de habla.

El objetivo del presente estudio es comparar parámetros cuantificables del habla, entre diferentes grupos de edad y género, en adultos normales chilenos. 


\section{Método}

\section{Sujetos}

Para la realización de este estudio se seleccionó por conveniencia 90 adultos chilenos residentes de la Región Metropolitana, hablantes nativos del español, entre 40-69 años $(x=54.73)$ distribuidos en tres grupos de edad: a) grupo $1=40-49$ años $(x=45.57), 15$ hombres $(H)$ y 15 mujeres $(M) ; b)$ grupo $2=50-59$ años $(x=54.50) ; 15$ H, 15 M y c) grupo 3=60-69 años ( $\mathrm{x}=64.13) ; 16$ $\mathrm{H}, 14 \mathrm{M}$, todos con escolaridad básica completa.

Los criterios de exclusión fueron: alteraciones auditivas y visuales incompatibles con la aplicación de la prueba, trastornos neurológicos, cognitivos, psiquiátricos, de comunicación y de aprendizaje.

Todos los participantes firmaron un consentimiento informado previo a su participación.

\section{Instrumento}

Se utilizó un protocolo de evaluación de habla, creado en una investigación previa ${ }^{5}$, el cual incluye: (1) tiempo máximo fonatorio (TMF), (2) movimientos orales mandibulares, labiales y linguales, (3) diadococinesias orales, (4) velocidad de lectura oraly (5) velocidad del habla automática.

El TMF consiste en la máxima cantidad de tiempo, en segundos (s.), en la cual el sujeto puede mantener la emisión del sonido solicitado, tras la realización de una inspiración profunda. Los sonidos evaluados fueron /s/, /a/ e /i/.
Los movimientos orales evaluados fueron ciclos de: apertura-cierre mandibular, protrusión-retrusión lingual, movimientos linguales laterales (ápice lingual a comisura), ascenso-descenso lingual, apertura-cierre labial y protrusión-retracción labial. Se incluyó además la repetición aislada de las sílabas /pa/, /ta/y/ka/. Se contabilizaron todas las producciones ejecutadas sin error en $5 \mathrm{~s}$.

Las diadococinesias orales fueron medidas mediante la cantidad de repeticiones sin error de la serie /pa-ta-ka/y las palabras /pitúko/, /petáka/, /botíka/, /bodéga/, /médiko/ y /málaga/; medidas en $5 \mathrm{~s}$.

La velocidad de lectura se obtuvo mediante el cálculo del tiempo en s. que el sujeto tardó en leer el texto "El abuelo", que consta de 101 palabras, posterior a lo cual se calculó el valor de la velocidad en palabras por minuto (ppm).

Finalmente, para medir la velocidad del habla automática se solicitó a los participantes que contasen de 1 a 20, determinándose la cantidad de segundos que tardó en la producción de la secuencia.

\section{Procedimiento}

La evaluación completa tuvo una duración aproximada de 30 minutos por sujeto. Primero se aplicaron los instrumentos de selección, correspondientes a un cuestionario de antecedentes, tanto demográficos como mórbidos, y el Mini Mental Test de Folstein (MMSE) ${ }^{6}$. Posteriormente se evaluaron los parámetros de 
habla en forma individual, en una sala libre de distractores, en condiciones de iluminación y temperatura adecuadas, donde se encontraba el participante y dos evaluadoras.

En cada evaluación se utilizó un cronómetro, una grabadora de audio digital (Olympus VN2100PC) y una cámara fotográfica digital (Cannon Power Shot SD 600) para el registro audiovisual del tercio inferior de la cara, posteriormente editado utilizando el programa Windows Movie Maker 2.18. Cada registro fue examinado por dos evaluadoras en forma independiente, certificando la confiabilidad interevaluador.

\section{Análisis estadístico}

Para el análisis estadístico de los resultados se utilizó el software Stata 10.1 (StataCorp. 2008. College Station, TX: StataCorp LP), expresando las variables en media y error estándar (ES).

Para la comparación de los datos según género se utilizó la prueba t para las variables paramétricas y el test de Mann-Whitney para las no paramétricas. Para el estudio comparativo de las variables según grupo de edad, se aplicó el análisis de varianza con método Bonferroni para las variables paramétricas y la prueba de Kruskal Wallis para las no paramétricas, considerándose como significativo un valor $\mathrm{p}<0.05$.

\section{Resultados}

A continuación se presentan los resultados comparativos por grupo de edad y género. En la Tabla 1 se presenta la comparación de los resultados obtenidos de acuerdo a los diferentes rangos de edad.

Los datos normativos resultantes de esta investigación revelan valores promedio de TMF de /s/ equivalentes a 19.37 s. en individuos de 4049 años de edad; 21.06 s. entre los 50-59 años y 18.61 s. entre los 60-69 años, mientras que el TMF de los sonidos /a/ e /i/ indican, respectivamente, 14.39 s y 18.77 s entre los 40-49 años, $16.56 \mathrm{~s}$ y 19.01 s entre $50-59$ años, 16.01 s y 18.44 s entre los 60-69 años.

Se esperaría que individuos entre $40 \mathrm{y}$ 49 años realizaran en 5 segundos un promedio de 11.85 ciclos de apertura-cierre mandibular; movimientos labiales equivalentes a 13.05 ciclos de apertura-cierre y 6.14 ciclos de protrusiónretrusión; 5.57 ciclos de protrusión-retrusión, 7.75 ciclos de lateralizaciones derecha-izquierda y 5.51 ciclos de ascenso-descenso linguales.

Entre los 50 y 59 años los ciclos de aperturacierre mandibular corresponden a 15.22 ciclos; los ciclos de movimientos labiales equivalen a 17.38 de apertura-cierre y 6.70 de protrusiónretrusión; mientras los linguales son de 5.73 ciclos de protrusión-retrusión, 8.93 de lateralizaciones derecha-izquierda y 6.25 de ascenso-descenso linguales. 
Tabla 1. Resultados promedios obtenidos de acuerdo a rango de edad

\begin{tabular}{|c|c|c|c|c|}
\hline Parámetro & $\begin{array}{c}\text { Grupo } 1 \\
\text { Media } \pm E S\end{array}$ & $\begin{array}{c}\text { Grupo } 2 \\
\text { Media } \pm E S\end{array}$ & $\begin{array}{c}\text { Grupo } 3 \\
\text { Media } \pm E S\end{array}$ & P Value \\
\hline \multicolumn{5}{|l|}{ Tiempo Máximo Fonación } \\
\hline TMF /s/ & $19.37 \pm 1.71$ & $21.06 \pm 1.84$ & $18.61 \pm 1.66$ & 0.701 \\
\hline TMF /a/ & $14.39 \pm 0.79$ & $16.56 \pm 1.34$ & $16.01 \pm 1.21$ & 0.554 \\
\hline TMF /i/ & $18.77 \pm 1.36$ & $19.01 \pm 1.40$ & $18.44 \pm 1.86$ & 0.635 \\
\hline \multicolumn{5}{|l|}{ Movimientos Orales } \\
\hline Apertura - cierre mandíbula & $11.85 \pm 0.76$ & $15.22 \pm 1.33$ & $13.02 \pm 0.98$ & 0.200 \\
\hline Apertura - cierre labios & $13.05 \pm 1.09$ & $17.38 \pm 1.32$ & $13.03 \pm 1.11$ & $0.033^{*}$ \\
\hline Protrusión - retrusión labios & $6.14 \pm 0.22$ & $6.70 \pm 0.28$ & $5.88 \pm 0.31$ & 0.080 \\
\hline Protrusión - retrusión lengua & $5.57 \pm 0.19$ & $5.73 \pm 0.28$ & $5.68 \pm 0.33$ & 0.957 \\
\hline Derecha - izquierda lengua & $7.75 \pm 0.46$ & $8.93 \pm 0.79$ & $7.48 \pm 0.40$ & 0.350 \\
\hline Ascenso - descenso lengua & $5.51 \pm 0.22$ & $6.25 \pm 0.31$ & $5.85 \pm 0.29$ & 0.256 \\
\hline \multicolumn{5}{|l|}{ Repetición de Sílabas } \\
\hline /pa/ & $32.73 \pm 0.77$ & $35.23 \pm 0.77$ & $31.30 \pm 0.92$ & $0.003 * *$ \\
\hline$/ \mathrm{ta} /$ & $33.27 \pm 1.16$ & $33.90 \pm 1.05$ & $32.13 \pm 0.73$ & 0.646 \\
\hline /ka/ & $29.90 \pm 1.03$ & $31.70 \pm 0.87$ & $29.37 \pm 0.83$ & 0.248 \\
\hline \multicolumn{5}{|l|}{ Diadococinesia } \\
\hline /pa-ta-ka/ & $10.94 \pm 0.33$ & $11.30 \pm 0.34$ & $10.90 \pm 0,25$ & 0.618 \\
\hline \multicolumn{5}{|l|}{ Repetición de Palabras } \\
\hline /pitúko/ & $11.83 \pm 0.27$ & $12.89 \pm 0.21$ & $12.01 \pm 0.30$ & $0.002 * *$ \\
\hline /petáka/ & $11.78 \pm 0.30$ & $12.57 \pm 0.25$ & $11.60 \pm 0.28$ & $0.004^{* *}$ \\
\hline /botíka/ & $11.63 \pm 0.28$ & $12.86 \pm 0.31$ & $11.63 \pm 0.32$ & $0.006^{* *}$ \\
\hline /bodéga/ & $11.84 \pm 0.30$ & $12.70 \pm 0.25$ & $11.63 \pm 0.36$ & $0.038^{*}$ \\
\hline /médiko/ & $11.33 \pm 0 ., 31$ & $12.21 \pm 0.27$ & $11.34 \pm 0.38$ & $0.027^{*}$ \\
\hline /málaga/ & $9.33 \pm 0.40$ & $10.11 \pm 0.25$ & $9.68 \pm 0.34$ & 0.206 \\
\hline Velocidad de Lectura & $150.76 \pm 4.73$ & $162.29 \pm 4,61$ & $159.35 \pm 3.16$ & 0.106 \\
\hline Velocidad de Habla Automática & $10.15 \pm 0.46$ & $8.60 \pm 0.41$ & $9.50 \pm 0.43$ & $0.011^{*}$ \\
\hline
\end{tabular}

* Diferencia estadísticamente significativa: $p<0,05$

** Diferencia estadísticamente significativa: $p<0,01$

Tiempo Máximo de Fonación en segundos; Movimientos Orales en número de ciclos en 5 segundos; Repetición de Sílabas en número de producciones sin error en 5 segundos; Diadococinesia en número de producciones sin error en 5 segundos; Repetición de Palabras en número de producciones sin error en 5 segundos; Velocidad de Lectura en palabras por minuto; Velocidad de Habla Automática en segundos para la producción de dígitos del 1 al 20.

A los 60-69 años, en tanto, se espera 13.02 ciclos de apertura-cierre mandibular; 13.03 ciclos de apertura-cierre y 5.88 de protrusión-retrusión labiales; 5.68, 7.48 y 5.85 ciclos de protrusiónretrusión, lateralizaciones derecha-izquierda y ascenso-descenso linguales, respectivamente.
Los valores normativos de repeticiones de sílabas medidas en cinco segundos corresponden a un número de 32.73 producciones de /pa/, 33.27 $\mathrm{de} / \mathrm{ta} /$ y 29.90 ejecuciones de $/ \mathrm{ka} /$ entre los 4049 años de edad, mientras estos valores alcanzan un promedio de $35.23,33.90$ y 31.70 repeticiones individuales de /pa/, /ta/ y / ka/ a los 50-59 años. 
Entre los 60-69 se espera un total de 31.30, 32.13 y 29.37 producciones de dichas sílabas, respectivamente.

Respecto a la repetición de las palabras / pitúko/, /petáka/, /botíka/, /bodéga/, /médiko/ y /málaga/, todas correspondientes a diadococinesias orales medidas en cinco segundos, la expectativa a los 40-49 años de edad es de 11.83, 11.78, 11.63, $11.84,11.33$ y 9.33 producciones; entre los 50-59 años es de 12.89, 12.57, 12.86, 12.70, 12.21 y 10.11 realizaciones, mientras a los 60-69 años se espera un promedio de 12.01, 11.60, 11.63, 11.63, $11.34 \mathrm{y}$ 9.68 repeticiones, respectivamente.

La norma obtenida para la valoración de la diadococinesia oral /pa-ta-ka/ medida en cinco segundos es de 10.94 producciones a los 40-49 años, 11.30 a los 50-59 años y 10.90 a los 60-69 años.

La velocidad de lectura esperada a los 40-49 años es de 150.76 ppm, mientras que a los 50-59 años equivale a 162.29 ppm y a los 60-69 años a $159.35 \mathrm{ppm}$.

Finalmente, la velocidad de habla automática entre los 40-49 años es de 10.15 segundos en la producción de la serie de dígitos de 1 a 20 , siendo este valor de 8.60 segundos a los 50-59 años y de 9.50 segundos a los 60-69 años.

Un análisis comparativo de los datos obtenidos muestra que en todos los parámetros evaluados, a excepción de velocidad del habla automática, el grupo 2 es el que realiza la mayor cantidad de ciclos por s. o tiempo de producción, de acuerdo a la tarea solicitada.

En el parámetro TMF todos los grupos obtuvieron un mayor valor en la /s/ y el menor en la /a/; no existen diferencias significativas en este parámetro al comparar por edad.

En relación a los movimientos orales, el único parámetro que presenta diferencias significativas es apertura y cierre de labios, que además corresponde al parámetro que se ejecuta con mayor velocidad en todos los grupos.

En repetición de sílabas y palabras existen diferencias significativas en la sílaba /pa/y palabras /pitúko/, /petáka/, /botíka/, /bodéga/ y /médiko/.

En diadococinesias orales y velocidad de lectura no existe diferencia entre los grupos.

Finalmente, en velocidad de habla automática sí existe diferencia estadísticamente significativa.

El análisis post hoc indica que para el parámetro apertura y cierre de labios el grupo 2 realizó más ciclos que el grupo 1.

En repetición de /pa/, /botíka/ y /bodéga/ el grupo 2 realiza más repeticiones que el grupo 3, mientras que para la repetición de /pitúko/, /petáka/ y /médiko/ el grupo 2 realiza más repeticiones que los grupos 1 y 3 .

En tanto, en la velocidad de habla automática el grupo 1 presenta un mayor rendimiento que el grupo 2 
Tabla 2. Resultados comparativos según género.

\begin{tabular}{|c|c|c|c|}
\hline Parámetro & $\begin{array}{l}\text { Hombres } \\
\text { Media } \pm E S\end{array}$ & $\begin{array}{l}\text { Mujeres } \\
\text { Media } \pm \text { ES }\end{array}$ & P Value \\
\hline \multicolumn{4}{|l|}{ Tiempo Máximo Fonación } \\
\hline $\mathrm{TMF} / \mathrm{s} /$ & $21.46 \pm 1.36$ & $17.98 \pm 1.43$ & $0.021^{*}$ \\
\hline $\mathrm{TMF} / \mathrm{a} /$ & $16.30 \pm 0.39$ & $15.03 \pm 0.93$ & 0.165 \\
\hline TMF /i/ & $20.13 \pm 1.37$ & $17.41 \pm 1.12$ & 0.153 \\
\hline \multicolumn{4}{|l|}{ Movimientos Orales } \\
\hline Apertura - cierre mandíbula & $13.13 \pm 0.82$ & $13.59 \pm 0 ., 92$ & 0.880 \\
\hline Apertura - cierre labios & $14.58 \pm 1.00$ & $14.40 \pm 1.01$ & 0.810 \\
\hline Protrusión - retrusión labios & $6.19 \pm 0.23$ & $6.29 \pm 0.23$ & 0.680 \\
\hline Protrusión - retrusión lengua & $6.07 \pm 0.23$ & $5.27 \pm 0.19$ & $0.005^{* *}$ \\
\hline Derecha - izquierda lengua & $8.21 \pm 0.54$ & $7.91 \pm 0.40$ & 0.580 \\
\hline Ascenso - descenso lengua & $5.98 \pm 0.20$ & $5.77 \pm 0.21$ & 0.400 \\
\hline \multicolumn{4}{|l|}{ Repetición de Sílabas } \\
\hline /pa/ & $34.05 \pm 0.66$ & $32.17 \pm 0.72$ & $0.031^{*}$ \\
\hline$/ \mathrm{ta} /$ & $33.66 \pm 0.95$ & $32.57 \pm 0.66$ & 0.174 \\
\hline /ka/ & $30.91 \pm 0.85$ & $29.76 \pm 0.65$ & 0.257 \\
\hline \multicolumn{4}{|l|}{ Diadococinesia } \\
\hline /pa-ta-ka/ & $11.23 \pm 0.28$ & $10.87 \pm 0.22$ & 0.157 \\
\hline \multicolumn{4}{|l|}{ Repetición de Palabras } \\
\hline /pitúko/ & $12.52 \pm 0.25$ & $11.98 \pm 0.19$ & 0.143 \\
\hline /petáka/ & $12.18 \pm 0.27$ & $11.79 \pm 0.20$ & 0.491 \\
\hline /botíka/ & $12.42 \pm 0.26$ & $11.68 \pm 0.25$ & $0.023^{*}$ \\
\hline /bodéga/ & $12.43 \pm 0.26$ & $11.70 \pm 0.24$ & $0.022^{*}$ \\
\hline /médiko/ & $11.85 \pm 0.27$ & $11.42 \pm 0.26$ & 0.515 \\
\hline /málaga/ & $9.94 \pm 0.32$ & $9.49 \pm 0.23$ & 0.106 \\
\hline Velocidad de lectura & $155.80 \pm 3.62$ & $159.06 \pm 3.38$ & 0.362 \\
\hline Velocidad de Habla Automática & $9.61 \pm 0.37$ & $9.23 \pm 0.36$ & 0.377 \\
\hline
\end{tabular}

* Diferencia estadísticamente significativa: $p<0,05$

** Diferencia estadísticamente significativa: $p<0,01$

Tiempo Máximo de Fonación en segundos; Movimientos Orales en número de ciclos en 5 segundos; Repetición de Sílabas en número de producciones sin error en 5 segundos; Diadococinesia en número de producciones sin error en 5 segundos; Repetición de Palabras en número de producciones sin error en 5 segundos; Velocidad de Lectura en palabras por minuto; Velocidad de Habla Automática en segundos para la producción de dígitos del 1 al 20.

En la Tabla 2 se presenta la comparación de los parámetros del habla según género. Como se observa, los hombres rinden mejor en todos los parámetros, excepto en apertura y cierre mandibular, protrusión y retrusión de labios y velocidad de lectura.
Sólo existen diferencias significativas en los parámetros TMF de /s/, protrusión y retrusión lingual, repetición de /pa/y repetición de palabras /botíka/ y /bodéga/, siendo los valores de estos parámetros mayores en el grupo de hombres. 


\section{Discusión}

El propósito del estudio fue comparar el rendimiento frente a tareas de habla en adultos chilenos normales según edad y género.

A la fecha, en la literatura no existían datos normativos para caracterizar a la población chilena en relación a estos parámetros de habla y los escasos datos existentes se basan en sujetos de habla inglesa. Lo anterior es interesante de evaluar, considerando que el habla es influenciada por las variaciones estructurales fonéticas y morfosintácticas del idioma en que se produzca.

Los resultados obtenidos en el presente estudio muestran diferencias importantes en relación a los datos de investigaciones previas. Respecto al parámetro TMF, la duración de la producción del sonido /s/ en nuestro estudio fue de 21.4 s. en hombres y 17.9 s. en mujeres, mientras que valores previos fijaban una media mayor, siendo para hombres de 29.1 s. y 19.48 s. para mujeres ${ }^{7}$. Además, no se encontraron diferencias significativas al comparar el TMF de /s/ entre cada grupo de edad, siendo el promedio de 19.3 s. para el grupo 1, 21.06 para el grupo 2 y 18.61 s. para el grupo 3. Una investigación precedente basada en sujetos entre 8 y 88 años (promedio 27.7) determinó una media menor correspondiente a 17.7 s. ${ }^{8}, \sin$ embargo, es importante considerar que las edades de ambas muestras no son comparables.

Respecto al TMF /a/, al comparar por género nuestro estudio obtuvo valores promedios de 16.3 s. para hombres y 15.0 s. para mujeres, lo que es similar a lo reportado en un estudio previo donde la media tanto para hombres y mujeres fue de 14.6 s. (edad entre 65-75 años y 66-93 años) 9. Por su parte, en el TMF/i/, en nuestro estudio encontramos valores de 20.1 s. en hombres y 17.4 s. en mujeres, lo que es mayor a lo reportado por $\mathrm{Kreul}^{9}$, donde la media para hombres fue de 16.8 s. y 14.8 s. para las mujeres, sin embargo, la edad promedio de esa muestra (69.8 años) es mayor a la de nuestro estudio (54.7 años).

En todos los estudios, incluido el nuestro, los sujetos presentan un mayor TMF de /s/, seguido de la /i/ y luego /a/; este resultado puede ser explicado debido a que la producción de la /s/, al ser fricativa, presenta una mayor resistencia a la salida del flujo del aire, permitiendo de esta forma prolongar por más tiempo el sonido. En contraste, el sonido /a/ es el que se produce con menor resistencia ${ }^{7}$. Por otro lado, en relación a la variable género, el que los hombres produzcan los mayores TMF, puede ser debido a que presentan una mayor capacidad vita ${ }^{10}$. La evaluación del TMF es fundamental, ya que la disminución en su duración puede revelar patrones alterados en el uso de la voz hablada, insuficiencia respiratoria, control glótico deficiente o alteraciones neurogénicas ${ }^{11}$.

En relación a los movimientos orales, tanto al comparar por género como por edad, la mayor cantidad de ciclos se produjo en apertura y cierre labial, seguido de apertura y cierre mandibular. Por su parte los movimientos linguales fueron los de menor ejecución. Resulta difícil explicar las razones de estas diferencias. El movimiento de 
ascenso y descenso lingual solicitado consistió en el contacto del ápice lingual con el bermellón del labio superior e inferior en forma alternada, en apertura mandibular. Lo anterior puede generar diferencias entre sujetos basadas no sólo en la tonicidad lingual, sino también en posibles restricciones de alcance debidas al punto de inserción y longitud del frenillo sublingual, así como la morfología facial y su influencia en las dimensiones verticales.

La morfología facial, el tipo de oclusión y la presencia de anomalías dentomaxilares no fueron considerados en este estudio, debido a que estas variaciones se encuentran presentes en la población general. Por otra parte los movimientos linguales requieren de un control más fino en comparación al descenso y ascenso mandibular, por lo que la precisión y coordinación de los movimientos es más compleja, especialmente al ejecutarse en series de movimientos. Los ciclos contabilizados en este estudio sólo fueron aquellos ejecutados respetando la amplitud de movimiento esperada.

Las fibras pertenecientes a la musculatura masticatoria, lingual y labial presentan diferente composición, lo cual repercute en su velocidad de contracción y fatigabilidad. Se sabe que los músculos elevadores de la mandíbula contienen gran cantidad de fibras híbridas, lo cual provee importantes propiedades contráctiles ${ }^{12}$. Estas fibras son más rápidas que otras formas puras encontradas en la porción posterior de la lengua, lo que podría explicar el menor número de ciclos observado en tareas de protrusión y retrusión lingual, sin embargo, son más lentas que otras encontradas en la musculatura labial ${ }^{13}$, en la cual predominan fibras puras que favorecen ejecuciones de alta intensidad. Estas últimas, sin embargo, son altamente fatigables ${ }^{14}$, lo que podría explicar el menor número de ciclos producidos al incrementar el tiempo de duración en tareas de apertura y cierre labial.

La apertura mandibular estaría comandada por fibras adaptadas para ejecutar movimientos rápidos y menos fatigables en comparación a las fibras encontradas en el orbicular. La musculatura lingual anterior, en tanto, posee fibras lentas, mientras la intrínseca presenta fibras híbridas; sin embargo, la contribución de estas últimas en los ejercicios evaluados es menor que la generada por la musculatura extrínseca, por lo que este factor no representa mayores diferencias en los resultados.

Debido a que las fibras con menor velocidad de contracción poseen menor fatigabilidad, probablemente en mediciones más prolongadas de estas tareas los movimientos linguales arrojarían resultados distintos.

En relación al parámetro repetición de sílabas, se obtuvo una media general de 33.1 ciclos en 5 s. para la repetición de /pa/, siendo mayor el promedio en los hombres y en el grupo de edad de 50-59 años, encontrándose diferencias significativas tanto por edad como por género.

Nuestros resultados son similares a los descritos por Ptacek ${ }^{15}$, quien encontró un mayor rendimiento en los hombres jóvenes (35.0 ciclos) 
que en las mujeres jóvenes (34.5 ciclos), y un rendimiento menor en la población adulta mayor (27.0 hombres y 25.0 mujeres).

Por otro lado, Kreul ${ }^{9}$ no encontró diferencias al comparar estas variables, siendo el rendimiento para ambos géneros similar entre el grupo de jóvenes y el de adultos mayores.

Respecto a la repetición de la sílaba /ta/ y $/ \mathrm{ka} /$, si bien no se encontraron diferencias tanto por edad como por género, nuestros resultados difieren a lo encontrado en estudios previos. Para la sílaba/ta/ el presente estudio obtuvo un promedio de 33.6 ciclos para hombres y 32.5 para las mujeres, lo que difiere de lo reportado en hombres ( 30 y 34.5 ciclos) y mujeres ( 29 y 34 ciclos) jóvenes ${ }^{9,15}$. En la sílaba /ka/, en nuestro estudio se obtuvieron 30.9 ciclos para hombres y 29.7 para mujeres, valores mayores a los descritos previamente ${ }^{9,15}$.

Respecto a las diadococinesias orales (/pa-ta-ka/), si bien no encontramos diferencias por edad y género, es importante destacar que los valores promedios obtenidos son menores a los descritos para el habla inglesa ${ }^{15}$. Sin embargo, la medición en este estudio fue realizada en $1 \mathrm{~s}$. versus los $5 \mathrm{~s}$. del nuestro, este mayor tiempo en la medición puede aumentar la exposición al error articulatorio, al igual que la probabilidad de generar una mayor fatigabilidad en los sujetos.

Finalmente, en relación al parámetro de velocidad del habla un estudio previo encontró que los sujetos jóvenes (21.7 años) producen 224.5 ppm y los adultos mayores (70 años) 156 ppm ${ }^{9}$.
En nuestro estudio, la velocidad del habla, según edad, fue de 150.7 ppm para el grupo 1, de 162.2 ppm para el grupo 2 y 159.3 ppm para el grupo 3, no observándose diferencia entre los grupos.

En ambos estudios podemos observar que el grupo de adultos mayores produce menor cantidad de palabras por minuto. Es sabido que con el envejecimiento se producen una serie de cambios a nivel estructural, en la movilidad, coordinación y sensibilidad, los cuales pueden lentificar los procesos de ejecución del habla ${ }^{16,17}$.

En conclusión, el presente estudio demuestra que existen diferencias en los parámetros de habla, tanto por género como por grupo de edad; estas diferencias se dan en los parámetros TMF, movimientos orales, repetición de sílabas, repetición de palabras y velocidad del habla automática. Los hombres rinden mejor que las mujeres y el grupo de edad de 50-59 años rinde mejor que los grupos de edad de 40-49 y 60-69 años. Por otro lado, los valores obtenidos en la presente investigación difieren de los descritos en estudios previos, realizados en otro idioma, lo que avala la necesidad de contar con datos normativos específicos para nuestra población. 


\section{Referencias}

1. Tasko, S. (2004) Variations in Articulatory Movement With Changes in Speech Task.J Speech Hear Res, Vol.47, 85-100.

2. Duffy, J. (2005) Motor Speech Disorders: Substrates, differential diagnosis and management. Philadelphia: Elsevier Mosby.

3. Instituto Nacional de Estadísticas INE (2007) Estadísticas vitales, Informe anual.

4. Lavados, P.M.; Sacks, C.; Prina, L.; Escobar, A.; Tossi, C.; Araya, F. \& cols. (2005) Incidence, 30-daycase-fatality-rate, and prognosis of stroke in Iquique, Chile: a 2-year community based prospective study (PISCIS proyect). Lancet, Vol. 365, 2206-2215.

5. Bahamonde, C.; González, J.; Martínez, M. \& Muñoz, M. (2007) Estandarización de parámetros cuantificables de habla en adultos normales Chilenos. Seminario de Investigación, Escuela de Fonoaudiología, Universidad de Chile.

6. Folstein, M.; Folstein, S.E. \& McHugh, P.R. (1975) Mini-Mental State a Practical Method for Grading the Cognitive State of Patients for the Clinician. J Psychiat Res, Vol.12, 189-198.

7. Pasewang, M. \& Pazera, J. (2006) Maximum duration of sustained /s/ and /z/. J Voice, Vol. 20, 369-379.

8. Eckel, F. \& Boone, D. (1981) The $s / z$ ratio as an indicator of laryngeal pathology. J Speech Hear Res, Vol.46, 147-149.
9. Kreul, E. (1972) Neuromuscular Control Examination (NMC) for Parkinsonism: Vowel Prolongations and Diadochokinetic and Reading Rates. J Speech Hear Res, Vol.15, 72-83.

10. Kent, R. (1987) Maximum Performance Test of Speech Production. J Speech Hear Res, Vol.52, 367-387.

11. Jackson-Menaldi, C. (2002) La voz patológica. Buenos Aires: Editorial Panamericana.

12. Korfage, J.; Koolstra, J.H.; Langenbach, G. \& Van Eijden, T. (2005) Fiber-type Composition of the Human Jaw Muscles (Part 1). Origin and Functional Significance of Fiber-type Diversity. J Dent Res, Vol. 84, 9, 774-783.

13. Kent, R.D. (2004) Development, Pathology and Remediation of Speech. From Sound to Sense: June 11-13, at MIT.

14. Scott, W.; Stevens, J. \& Binder, S.A. (2001) Human Skeletal Muscle Fiber Type Classifications. Phys Ther, 81:1810-1816.

15. Ptacek, P.; Sander, E. \& Maloney, W. (1966) Phonatory and Related Changes with Advanced Age. J Speech Hear Res, Vol.9, 353-360.

16. Rodríguez, R.; Camargos, C.; Antunes, T.; Fontes, L. \& Franco, V. (2009) Effects of the Aging Process on Respiratory Function. Gerontology, 55:505-510.

17. Ney, D.; Weiss, J.; Kind, A. \& Robbins, J. (2009) Senescent Swallowing: Impact, Strategies and Interventions. Nutr Clin Pract, 24: 395-413. 